\title{
Simulation of the seismic response of sedimentary basins with constant-gradient velocity along arbitrary direction using boundary element method: SH case*
}

\author{
Zengxi Ge* \\ Department of Geophysics, School of Earth and Space Sciences, Peking University, Beijing 100871, China
}

\begin{abstract}
We presented a boundary element method using the approximate analytical Green's function given by Sánchez-Sesma et al. Coordinate transform is introduced to extend the method to deal with the model with constant-gradient velocity along oblique direction. The method is validated by comparing the numerical results with other independent methods. This method provides a useful tool for analyzing local site effects. We computed seismic response for two series of models. The results in both frequency and time domains are analyzed and show complex amplification patterns. The fundamental mode of resonance is dependent not only on the velocity at the free surface but also on the velocity distribution of the whole basin. For the higher modes of vibration the heterogeneous basin also has its own characteristic.
\end{abstract}

Key words: boundary element; $\mathrm{SH}$ wave; seismic response; constant-gradient velocity

CLC number: P315.01 Document code: A

\section{Introduction}

Local effects due to sedimentary basins and topography can lead to significant temporal and spatial variations of seismic ground motions (See e.g., Aki, 1988; Geli et al, 1988; Bard, 1995; Sánchez-Sesma et al, 1996; Alvarez-Rubio et al, 2005). There are several ways of assessing site effects. The characterization of a given site can be achieved based on instrumental, theoretical-analytical and numerical approaches to the problem. A detailed survey of the numerical methods developed for the computation of site effects is given by Sánchez-Sesma (1996). The numerical methods that have been widely used to calculate the propagation of elastic waves inside sedimentary inclusions can be classified in domain, boundary, and asymptotic methods. In the first group, where the discretization of the media is required, are included techniques as the finite difference (Boore, 1972; Graves, 1996; Zhang and Chen, 2006), the finite element method (Smith, 1975; Bard, 1995;

\footnotetext{
${ }^{*}$ Received 16 October 2009; accepted in revised form 7 December 2009; published 10 February 2010.

• Corresponding author. e-mail: zge@pku.edu.cn

(c) The Seismological Society of China and Springer-Verlag Berlin Heidelberg 2010
}

Koketsu et al, 2004), pseudospectra method (Furumura et al, 1998; Wang et al, 2001) and spectra-element method (Komatitsch and Vilotte, 1998; Komatitsch and Tromp, 2002). On the other hand, in the boundary methods, where only the discretization of the boundaries is needed, there are two main approaches, one is based on the use of complete systems of solutions (Herrera, 1980), and the other on boundary integral equations (Brebbia, 1978). When the problem of the propagation of waves has an interest in the high frequency band and the diffraction can be ignored, the asymptotic methods (Cerveny and Hron, 1980; Cerveny and Brown, 2003) can be very useful. Among these techniques, boundary methods have some advantages over the domain methods in the cases where better accuracy is required near boundaries and sources, or where the domain needs to be extended to the infinity. Boundary methods require discretization of the model only on boundaries. This is advantageous for models composed of a number of homogeneous regions separated by sharp boundaries. A recent comprehensive review of these methods in dynamic elasticity is that of Bouchon and Sánchez-Sesma (2007). Since the analytical Green's function is required in fulfillment of the boundary methods, the availability 
of the Green's function limits the application of boundary element methods. Normally, only the Green's function for a homogeneous unbounded medium can be obtained.

There is a wide class of problems for which it is reasonable to assume an increase of wave propagation velocities with depth (see e.g, Bard and Gariel, 1986; Vrettos, 1990, 1991). An approximate analytic Green's function for 2-D case is given and tested by Sánchez-Sesma et al (2001), which allows us to extend the boundary element method to the medium with vertically increasing velocity. There are two main approaches for the boundary methods. (1) In the direct formulation of boundary integral equation methods (BIEM) one finds the unknown tractions and displacements at the boundaries of the domains, which was called BEM (for Boundary Element Method) by Brebbia (1978) and is used extensively since then, (2) While in the indirect approach one formulates the problem in terms of force or moment boundary densities, which was terminated as IBEM (Indirect boundary element method) by (Luco et al, 1990) and used thereafter. Luzón et al ( 2003, 2004) applied the approximate Green's function to the second approach and studied the seismic response of semi-circular basin. In this paper we applied this Green's function to the first approach (BEM) and analyzed the ground motion in sedimentary inclusions with velocity increasing along an oblique direction. We illustrated the effects of these sedimentary basins by means of frequency-space diagrams, synthetic seismograms on the free surface on the vertical under SH plane wave incidence. The effects of velocity gradient and obliquity on fundamental mode were also studied.

\section{Configuration of the problem}

The problem considered in this study is a 2-D alluvial valley on top of a half space, as shown in Figure 1. The basin is named region $R_{\mathrm{i}}$ and the half-space is called $R_{\mathrm{e}}$. The shear wave velocity and the density in the basin are assumed to increase along an oblique direction $z^{\prime}$ and can be expressed as (Sánchez-Sesma et al, 2001):

$$
\begin{gathered}
\beta\left(z^{\prime}\right)=\beta(0)\left(1+\gamma z^{\prime}\right), \\
\rho\left(z^{\prime}\right)=\rho(0)\left(1+\gamma z^{\prime}\right)^{n},
\end{gathered}
$$

where $\beta(0)$ and $\rho(0)$ are the velocity and density at $z^{\prime}=0$, respectively. $\gamma$ is the gradient of seismic wave velocity along $z^{\prime}$ direction and $n \geq 0$.

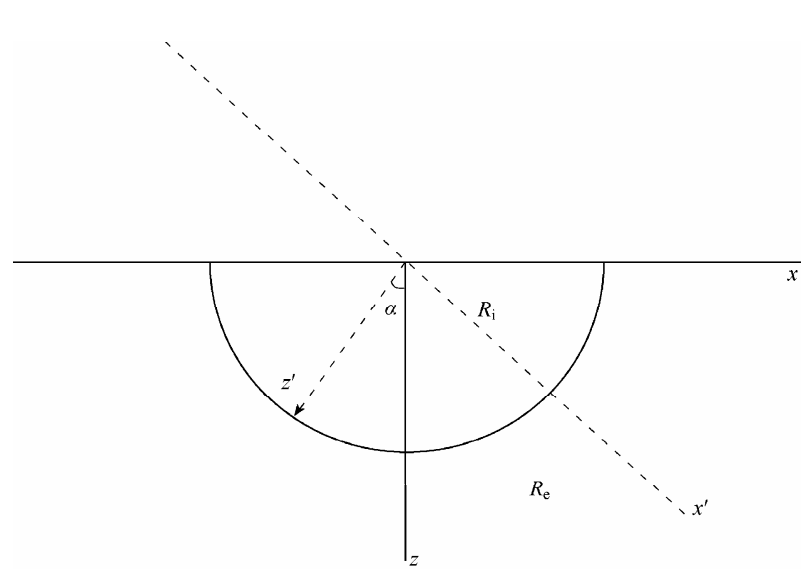

Figure 1 Configuration of the problem considered in this study. The basin is named region $R_{\mathrm{i}}$ and the half-space is called $R_{\mathrm{e}}$. The wave velocity in the basin is assumed to increase linearly along $z^{\prime}$. $\alpha$ denotes the angle between $z^{\prime}$ and $z$.

The boundary element method (BEM) is applied to calculating the seismic response of this configuration. The BEM is a numerical technique based on Somigliana's integral representation theorem in elasto-dynamics. Assuming harmonic excitation, the displacement inside the basin Ri can be expressed as (Brebbia, 1978)

$$
C(\boldsymbol{r}) u(\boldsymbol{r})+\int_{\Gamma_{\mathrm{i}}}\left[T\left(\boldsymbol{r}, \boldsymbol{r}^{\prime}\right) u\left(\boldsymbol{r}^{\prime}\right)-G\left(\boldsymbol{r}, \boldsymbol{r}^{\prime}\right) t\left(\boldsymbol{r}^{\prime}\right)\right] \mathrm{d} S\left(\boldsymbol{r}^{\prime}\right)=0,
$$

where $G\left(\boldsymbol{r}, \boldsymbol{r}^{\prime}\right)$ is the displacement Green's function and $T\left(\boldsymbol{r}, \boldsymbol{r}^{\prime}\right)=\mu \frac{\partial}{\partial n} G\left(\boldsymbol{r}, \boldsymbol{r}^{\prime}\right)$ is the traction Green's function. $\hat{\boldsymbol{n}}$ is normal direction of the surface.

$$
C(\boldsymbol{r})=\left\{\begin{array}{cc}
1 & \boldsymbol{r} \in R_{i} \\
\frac{1}{2} & \boldsymbol{r} \in \Gamma \\
0 & \boldsymbol{r} \notin R_{i}
\end{array}\right.
$$

In the half space $R_{\mathrm{e}}$,

$$
\begin{gathered}
C(\boldsymbol{r}) u(\boldsymbol{r})+ \\
\int_{\Gamma_{\mathrm{e}}}\left[T\left(\boldsymbol{r}, \boldsymbol{r}^{\prime}\right) u\left(\boldsymbol{r}^{\prime}\right)-G\left(\boldsymbol{r}, \boldsymbol{r}^{\prime}\right) t\left(\boldsymbol{r}^{\prime}\right)\right] \mathrm{d} S\left(\boldsymbol{r}^{\prime}\right)=u_{0}(\boldsymbol{r})
\end{gathered}
$$

where $u_{0}(\boldsymbol{r})$ denotes the incident wave field. To obtain the displacement on the boundary, the traction free condition along the free surface

$$
t(\boldsymbol{r})=0, \quad \boldsymbol{r} \in \Gamma_{\text {free surface }}
$$

and the continuity conditions of displacements and tractions along the interface 


$$
\left\{\begin{array}{c}
u_{1}=u_{2} \\
\mu_{1} \frac{\partial u_{1}}{\partial n}=\mu_{2} \frac{\partial u_{2}}{\partial n}
\end{array}\right.
$$

must be satisfied.

Equations (4), (5), (6) and (7) form a linear system of integral equations for the boundary sources. The displacement and tractions along the surfaces can be obtained by solving the linear equations.

The Green's functions in a homogeneous isotropic unbounded medium is given by (Brebbia, 1978),

$$
G\left(\boldsymbol{r}, \boldsymbol{r}^{\prime}, \omega\right)=-\frac{\mathrm{i}}{4 \mu} \mathrm{H}_{0}^{(2)}(k \boldsymbol{r}) .
$$

and the traction Green's function is

$$
T\left(\boldsymbol{r}, \boldsymbol{r}^{\prime}, \omega\right)=\mu \frac{\partial G}{\partial n}=\frac{\mathrm{i} k}{4} \mathrm{H}_{1}^{(2)}(k r) \hat{\boldsymbol{r}} \cdot \hat{\boldsymbol{n}},
$$

where $\mathrm{i}$ is the imagary number unit, $\mu$ is shear modulus, $k=\omega / \beta, \mathrm{H}_{n}^{(2)}=$ Hankel function of the second kind and the $n$th order, $\omega$ is circular frequency, $r=\left|\boldsymbol{r}-\boldsymbol{r}^{\prime}\right|=$ the distance between the source and receiver, $\hat{\boldsymbol{r}}=\boldsymbol{r}-\boldsymbol{r}^{\prime}|| \boldsymbol{r}-\boldsymbol{r}^{\prime} \mid$. $\beta=$ the shear wave velocity.

In the sedimentary basin with velocity increasing linearly with depth, Sánchez-Semsma et al (2001) presented an approximate analytical expression based on the asymptotic ray theory and accounted for both near-source effects and low frequencies. Luzón et al. (2009) improved the formulae and validated the Green's function using an accuracy analysis to compute the relative error in frequency domain for the SH case, and with a quantitative analysis obtaining envelope and phase misfits of the solution in time domain, for the P-SV case Here we just summarize the Green's function for SH case according to Luzón et al (2009). The displacement Green's function for earth model described by equations (1) and (2) can be expressed as

$$
G\left(\boldsymbol{r}^{\prime}, \boldsymbol{r}_{0}^{\prime}, \omega\right)=\Lambda \frac{1}{4 \mu_{0} i} \mathrm{H}_{0}^{(2)}(\omega \tau)
$$

where

$$
\Lambda=\left(\frac{1+\gamma z_{0}^{\prime}}{1+\gamma z^{\prime}}\right)^{\frac{n}{2}+1} \sqrt{2 \ln \left(\frac{R_{2}+R_{1}}{R_{2}-R_{1}}\right) \frac{\left(z_{0}^{\prime}+h\right)\left(z^{\prime}+h\right)}{R_{1} R_{2}}}
$$

with

$$
\begin{gathered}
R_{1}=\sqrt{\left(x^{\prime}-x_{0}^{\prime}\right)^{2}+\left(z^{\prime}-z_{0}^{\prime}\right)^{2}}, \\
R_{2}=\sqrt{\left(x^{\prime}-x_{0}^{\prime}\right)^{2}+\left(z^{\prime}+z_{0}^{\prime}+2 h\right)^{2}} .
\end{gathered}
$$

$$
\tau=h /\left[\beta(0) \ln \left(\frac{R_{2}+R_{1}}{R_{2}-R_{1}}\right)\right] \text { is the travel time. It }
$$

can be shown that when $h \rightarrow \infty$, equation (10) becomes the Green's function in homogenous case as shown in equation (8). To compute the Green's function in $x-z$ system, we need to transfer the location of the source and receivers to the $x^{\prime}-y$ ' system by:

$$
\left\{\begin{array}{c}
x^{\prime}=x \cos \alpha+z \sin \alpha \\
z^{\prime}=-x \sin \alpha+z \cos \alpha
\end{array}\right.
$$

Then we can obtain the Green's function under $x^{\prime}, y^{\prime}$ coordinates.

\section{Validation of the numerical approach}

In order to test this numerical approach, we compare our results with published results by others for two cases. The first one is the homogenous case which is the limit case with $\gamma=0$ in equation (10), and the second one is the case in which the velocity of the basin increases with depth. The problem considered is the seismic response of a semi-circular basin under the incident $\mathrm{SH}$ waves, which has been computed for the normalized frequency $\eta=2 a / \lambda=a \omega /\left(\pi \beta_{\mathrm{e}}\right)=1.0$, with $a$ being the radius of the sedimentary basin, and $\beta_{\mathrm{e}}$ shear wave velocity in the half space. The physical properties of the basin in the first case shown in Figure 1 are $\mathrm{S}$-wave velocity $\beta_{\mathrm{i}}=\beta_{\mathrm{e}} / 2=1.0 \mathrm{~km} / \mathrm{s}$, and a density of $\rho_{\mathrm{i}}=2 \rho_{\mathrm{e}} / 3=1.0 \mathrm{~g} / \mathrm{cm}^{3}$. Figure 2 displays the surface displacements for incident SH plane waves with the incident angle of $30^{\circ}$. In the same plot the exact solution provided by (Trifunac, 1971) and the results of Sánchez-Sesma et al (1993) using the indirect boundary element method are shown in solid triangles and circles, respectively. The agreement among three results is excellent.

In the second case the material properties considered are $\beta_{\mathrm{i}}(0)=\beta_{\mathrm{e}} / 3, \gamma=1.0$ in equation (1), and a constant density of $\rho_{\mathrm{i}}=2 \rho_{\mathrm{e}} / 3$ [n=0 of equation (2)]. Figure 3 shows the amplitude of displacement along surface for normalized frequency $\eta=1$ caused by vertical incident SH plane waves calculated by our method (solid line), thin line computed by Luzón et al (2004) and those symbols computed by Benites and Aki (1994) using a boundary integral-Gaussian beam method, and those computed by the same authors with finite element method. The overall agreement of the four results obtained independently is pretty good, although there is some difference in the middle of the basin. The com- 
parison shows the validation of our method. The boundary integral-Gaussian beam method uses Gaussian beam as the Green's function for both the basin and the half space while Luzón et al (2004) present method using exact Green's function in the half space and approximate

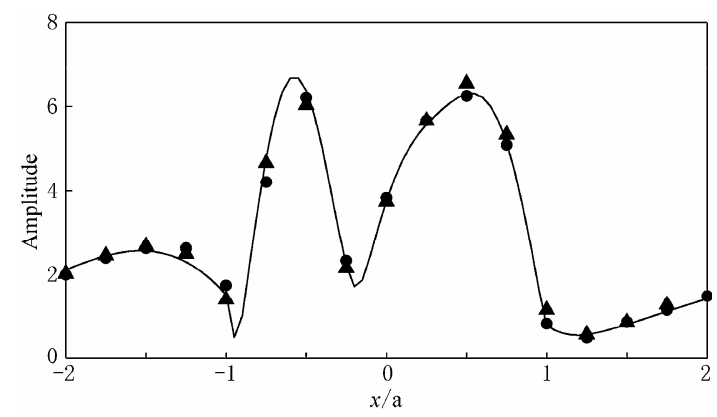

Figure 2 Amplitudes of displacement for incident $\mathrm{SH}$ plane waves with $30^{\circ}$ incident angle upon a homogeneous semi-circular sedimentary inclusion. Normalized frequency $\eta=1$. Solid line corresponds to our results, while solid triangles and circles correspond to the solution of Sánchez-Sesma et al (1993) and Trifunac (1971), respectively.

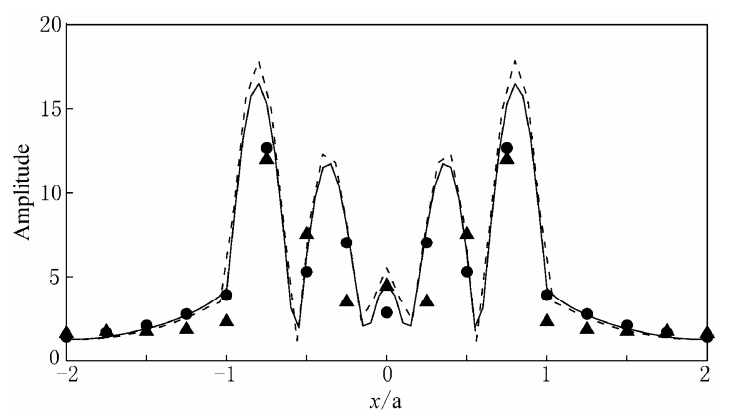

Figure 3 Amplitudes of SH displacement for vertical incident $\mathrm{SH}$ plane waves upon a semi-circular sedimentary basin with velocity increasing linearly with depth. Normalized frequency $\eta=1$. Solid line corresponds to our results, while dashed line correspond to the solution provided by Luzón et al (2004) using IBEM. Solid triangles and circles correspond to the results of Benites and Aki (1994) using a boundary integral-Gaussian beam method and the finite element method, respectively.

analytical Green's function in the basin. The finite element method divides the space into small elements with homogenous velocity parameters. Since the Gaussian beam is high-frequency approximation of the wave field, which may lead to the big difference of its results out of the basin to other three results. Furthermore, the homogenous velocity in the elements used in the finite difference method is the main reason for its notable difference in the middle of the basin.

\section{Numerical examples}

In order to show the basic characteristic of the displacement amplification by the sedimentary basin with different shear wave velocity profiles, we computed the seismic response of three different models including one homogenous basin model and two heterogeneous basin models. In all cases, the basin has a semi-circular shape with radium of $1 \mathrm{~km}$. The model used in the boundary element method has a horizontal range of -10 through $10 \mathrm{~km}$. The density and velocity for the half space of all these models are set to $\rho_{\mathrm{e}}=1.5 \mathrm{~g} / \mathrm{cm}^{3}, \beta_{\mathrm{e}}=3.0 \mathrm{~km} / \mathrm{s}$. In the basin, the density are all set to $\rho_{\mathrm{i}}=1.0 \mathrm{~g} / \mathrm{cm}^{3}$, which mean $m=0$ in equation (2). For heterogeneous models, the minimum values are $1.0 \mathrm{~km} / \mathrm{s}$ which appears at $x$ of $1 \mathrm{~km}$ while the maximum velocities are $2.0 \mathrm{~km} / \mathrm{s}$, which emerges at $z^{\prime}$ of $1 \mathrm{~km}$. The velocity dependences with the position are shown in Table 1 and velocity profiles are shown in Figure 4a.

Table 1 Density and velocity profiles for three different models

\begin{tabular}{c|clc}
\hline Model & Density & \multicolumn{1}{c}{ velocity } & obliquity \\
\hline$M_{1}$ & $\rho_{\mathrm{i}}=1.0 \mathrm{~g} / \mathrm{cm}^{3}$ & $\beta_{\mathrm{i}}(z)=1.0 \mathrm{~km} / \mathrm{s}$ & - \\
$M_{2}$ & $\rho_{\mathrm{i}}=1.0 \mathrm{~g} / \mathrm{cm}^{3}$ & $\beta_{\mathrm{i}}(z)=1.0+1.0 z \mathrm{~km} / \mathrm{s}$ & $\alpha=0^{\circ}$ \\
$M_{3}$ & $\rho_{\mathrm{i}}=1.0 \mathrm{~g} / \mathrm{cm}^{3}$ & $\beta_{\mathrm{i}}\left(z^{\prime}\right)=1.3333+0.6667 z^{\prime} \mathrm{km} / \mathrm{s}$ & $\alpha=30^{\circ}$ \\
\hline
\end{tabular}

We computed the displacement amplitude in the frequency domain from 0.05 to $5.0 \mathrm{~Hz}$ and for 81 receivers along the free surface from -2 to $2 \mathrm{~km}$. The time required for each model for 120 frequencies is about five minutes. The results in the frequency-space $(f-x)$ domain are shown in Figure $4 \mathrm{~b}$ and the synthetic seismograms shown in Figure $4 \mathrm{c}$ are generated by convoluting a Ricker wavelet (Ricker, 1953) with central frequency of $1.25 \mathrm{~Hz}$. Similar to the results of Luzón et al (2004), these three models produced significantly different patterns in both $f-x$ domain and time domain. For all these models, high-impedance contrast makes the surface waves reflected back and forth at the edges of the basin, producing well-defined resonances. However, the frequency, location and the amplification factors are different. For $M_{1}$, the frequency of the fundamental mode is about $0.37 \mathrm{~Hz}$ and the maximum amplification occurs in the middle of the basin for most modes. For heterogeneous model $M_{2}$, the frequency of the fundamental mode is about $0.5 \mathrm{~Hz}$, due to higher average velocity. For the higher modes, this model has its own characteristic. The locations of maximum amplification for the higher modes are not in the middle of the basin. For model $M_{3}$, the maximum appli- 
cation of the modes are no longer in the middle of the basin, it moves to the right instead. For models $M_{1}$ and $M_{2}$, the displacement in the basin composed by the interferences between the different waves rebounding up and down and the surface waves that propagate back and forth In contrast, at the receivers located on the half-space, the amplitude is much smaller and energy refracted from the basin can be clearly seen. For model $M_{3}$, the vibration in the right corner of the basin lasts longer and the amplitude is larger than the left part. Since the left part has lower impendence contrast, more energy leaks out to the half-space.
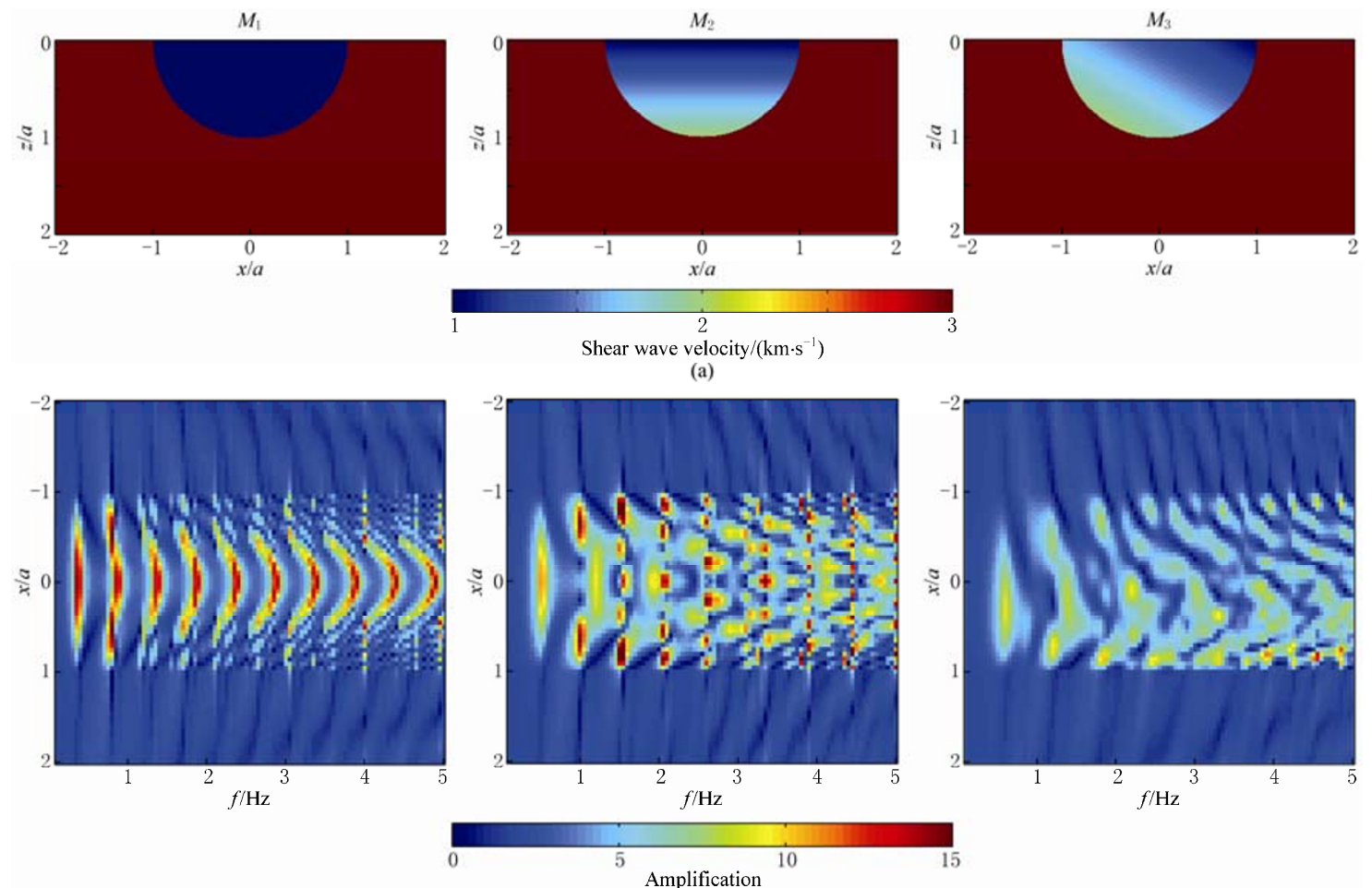

(b)
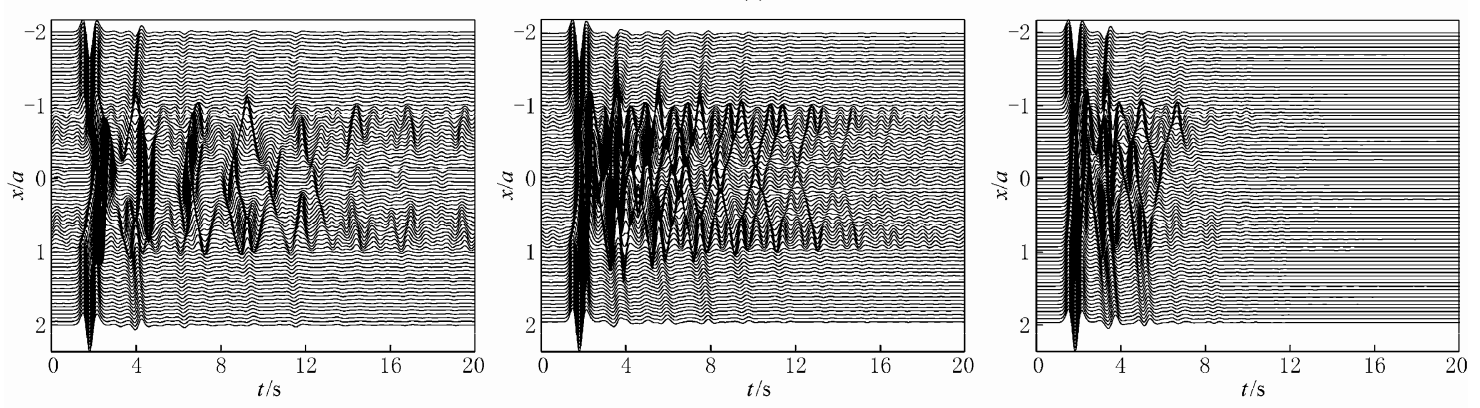

(c)

Figure 4 Velocity profiles for three models (a); Amplitudes of the displacement at the free surface in the frequency-space domain $(f-x)(b)$; Vertical incidence of SH waves upon each of the three sedimentary basins (c), synthetic seismograms of the displacement at the free surface of the three models under vertical incidence of SH waves. The source time function is a Ricker wavelet with a central frequency of $1.25 \mathrm{~Hz}$.

In order to show the influence of the velocity gradient and obliquity, we computed two sets of models and picked out the location, frequency and maximum amplification factors for models with different gradients and obliquity. First, the obliquity is fixed to $\alpha=0$, which means the velocity increase vertically. The responses in frequency domain for 20 different gradients from 0.1 to 2.0 are computed. In these cases, the maximum amplification locates at the middle of the basin. As shown in Figure 5, as the velocity gradients increases the resonant frequency increases linearly from 0.38 to $0.88 \mathrm{~Hz}$ due to the linearly increasing average velocity. At the same 
time, since the impedance contrast decreases, the amplification factor decreases exponentially. For second group of models, we fix the average velocity on the surface of the basin as $1.0 \mathrm{~km} / \mathrm{s}$ and the velocity gradient as $1.0 \mathrm{~s}^{-1}$, and computer 14 models with the oblique angle increases from $5^{\circ}$ to $70^{\circ}$. As the obliquity increases, the velocity in the right corner gets smaller. As shown in Figure 6, as the angle increases, the location of the maximum moves to the right and the maximum resonant frequency increase from 0.44 to $0.6 \mathrm{~Hz}$. At the same time, the amplification factors increase exponentially since the impedance contrast increases in the right corner of the basin. From these two results, we can see that both the gradient and the obliquity can affect the seismic response of the basin. In order to estimate the ground motion of earthquakes, not only the velocity at the surface but also the real subsurface structure are required.
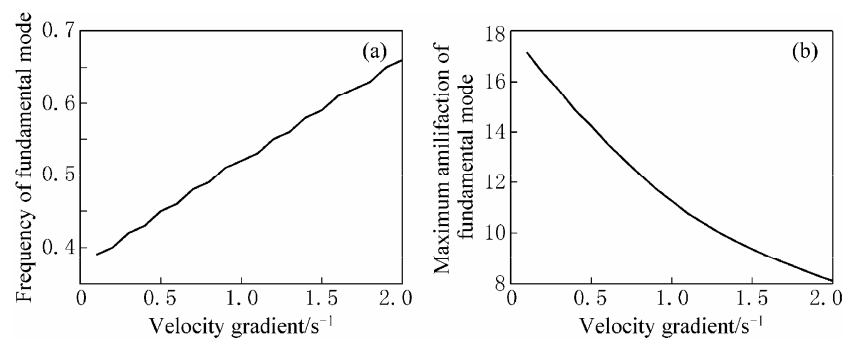

Figure 5 Resonant frequency (a) and maximum amplifaction factor of the fundamental mode (b) for models with different verticle velocity gradient.
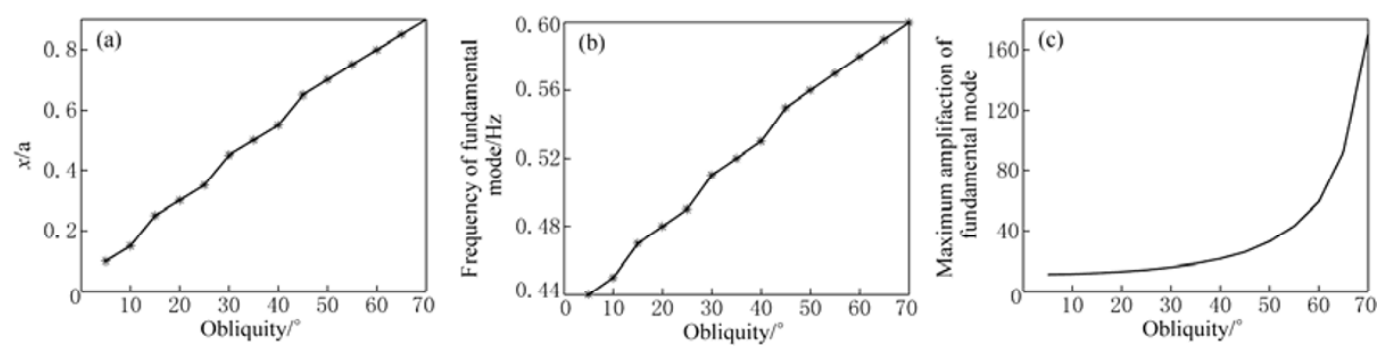

Figure 6 Location (a), resonant frequency (b) and maximum amplifaction of fundamental mode (c) for models with different oblique angle.

\section{Discussion and conclusions}

We presented a boundary element method (BEM) using the approximate analytical Green's function given by Sánchez-Sesma et al (2001). Coordinate transform is introduced to extend the method to deal with the model with constant-gradient velocity along oblique direction. The method is validated by comparing the numerical results with the other independent methods. This method provides a useful tool for analyzing the local site effects. Although we limited our examples to the model with $\mathrm{S}$ wave velocity increasing along an oblique direction in a semi-circular basin, the shape of the basin can be arbitrary and the velocity in the half-space can also be heterogeneous. More comprehensive analysis for all these models is the direction of our further work.

As an example, we have computed the seismic response of homogeneous and heterogeneous models of sedimentary basins above a half-space using this method for vertical incidence of SH plane waves. The case of incident $\mathrm{P}$ and $\mathrm{SV}$ waves can be easily treated using the appropriate Green's functions following the same procedure. The results in both frequency and time domains are analyzed and show complex amplification patterns. The fundamental mode of resonance depends not only on the velocity along the free surface, but also on the velocity distribution of the whole basin. For the higher modes of vibration the heterogeneous basin has its own characteristic.

Acknowledgements We are grateful to Dr. Sánchez-Sesma for proving the Green's function and useful discussions with him. This work is jointly supported by the National Science Foundation of China (Nos. D40444002 and D40521002) and National Key Basic Research Program (No.2006CB705803).

\section{References}

Aki K (1988). Local site effects on strong ground motion.In: Lawrence Von Thun J ed. Earthquake Engineering and Soil Dynamics. II. Recent Advances in Ground Motion Evaluation. Geotechnical Special Publication No. 20, 27-30.

Alvarez-Rubio S, Benito J J, Sánchez-Sesma F J and Alarcón E (2005). The use of direct boundary element method for gaining insight into complex seismic site response. Computers and Structures 83: 821-835.

Bard P and Gariel J (1986). The seismic response of two-dimensional sedimentary deposits with large vertical velocity gradients. Bull Seism Soc Amer 76: 343.

Bard P Y (1995). Effects of surface geology on ground motion: Recent results and remaining issues. In: Proceeding of the 10th European Conference on 
Earthquake Engineering. European Association on Earthquake Engineering, Vienna, 305-323.

Benites R and Aki K (1994). Ground motion at mountains and sedimentary basins with vertical seismic velocity gradient. Geophys J Int 116: 95-118.

Boore D (1972). A note on the effect of simple topography on seismic SH waves. Bull Seism Soc Amer 62: 275.

Bouchon M and Sánchez-sesma F J (2007). 'Boundary integral equations and boundary elements methods in elastodynamics', Advances in geophysics, $48,157-89$.

Brebbia C (1978). Recent Advances in Boundary Element Methods. Pentech Press, London.

Cerveny V and Brown M (2003). Seismic ray theory. The Journal of the Acoustical Society of America 113: 14.

Cerveny V and Hron F (1980). The ray series method and dynamic ray tracing systems for 3D inhomogeneous media. Bull Seim Soc Amer 70: 47-77.

Furumura T, Kennett B and Takenaka H (1998). Parallel 3-D pseudospectral simulation of seismic wave propagation. Geophysics 63: 279 .

Geli L, Bard P Y and Jullien B (1988). The effect of topography on earthquake ground motion: A review and new results. Bull Seism Soc Amer 78: 42-63.

Graves R (1996). Simulating seismic wave propagation in 3D elastic media using staggered-grid finite differences. Bull Seism Soc Amer 86: 1091.

Herrera I (1980). Boundary methods: A criterion for completeness. In: Proceeding of National Academic of Science of USA. 77: 4395-4398.

Koketsu K, Fujiwara H and Ikegami Y (2004). Finite-element simulation of seismic ground motion with a voxel mesh. Pure Appl Geophys 161: 2183-2 198.

Komatitsch D and Tromp J (2002). Spectral-element simulations of global seismic wave propagation. I. Validation. Geophys J Int 149: 390-412.

Komatitsch D and Vilotte J (1998). The spectral element method: an efficient tool to simulate the seismic response of $2 \mathrm{D}$ and $3 \mathrm{D}$ geological structures. Bull Seism Soc Amer 88: 368-392.

Luco J, Wong H and De Barros F (1990). Three-dimensional response of a cylindrical canyon in a layered half-space. Earthq Eng Struc Dynam 19: 799-817.

Luzón F, Ram rez L, Sánchez-Sesma F and Posadas A (2003). Propagation of SH elastic waves in deep sedimentary basins with an oblique velocity gradient. Wave Motion 38: 11-23.

Luzón F, Ramírez L, Sánchez-Sesma F J and Posadas A (2004). Simulation of the seismic response of sedimentary basins with vertical constant-gradient velocity for incident SH waves. Pure Appl Geophys 161: 1533-1 547.
Luzón F, Sánchez-sesma F, Pérez-Ruiz A, Ramírez-Guzman L and Pech A (2009). In-plane seismic response of inhomogeneous alluvial valleys with vertical gradients of velocities and constant Poisson ratio. Soil Dynam Earthq Eng 29(6): 994-1 004.

Ricker N (1953). Wavelet contraction, wavelet expansion, and the control of seismic resolution. Geophysics 18: 769 .

Sánchez-Sesma F J (1996). Strong ground motion and site effects. 9. In: De Beskos DE, Anagnostopoulos SA, editors. Computer analysis and design of earthquake resistant structures. Southampton: Computational mechanics publications, 201-239.

Sánchez-Sesma F J, Bentes,R. and Bielak J (1996). The assessment of strong ground motion: What lies ahead. In: Proceedings of the 11th World Conference on Earthquake Engineering. Acapulco, Mexico, Paper No. 2014.

Sánchez-Sesma F J, Madariaga R and Irikura K (2001). An approximate elastic two-dimensional Green's function for a constant-gradient medium. Geophys J Int 146: 237-248.

Sánchez-Sesma F J, Ramos-Martinez J and Campillo M (1993). An indirect boundary element method applied to simulate the seismic response of alluvial valleys for incident P, S and Rayleigh waves. Earthq Eng Struc Dynam 22: 279-295.

Smith W (1975). The application of finite element analysis to body wave propagation problems. Geophys $J$ R astr Soc 42: 747-768.

Trifunac M D (1971). Surface motion of a semi-cylindrical alluvial valley for incident plane SH waves. Bull Seism Soc Amer 61: 1 755-1 770.

Vrettos C (1990). Dispersive SH-surface waves in soil deposits of variable shear modulus $=$ Ondes de surface $\mathrm{SH}$ dispersives dans un sol de module de cisaillement variable. Soil Dynam Earthq Eng 9: 255-264.

Vrettos C (1991). Time-harmonic Boussinesq problem for a continuously non-homogeneous soil. Earthq Eng Struc Dynam 20: 961-977.

Wang Y, Takenaka H and Furumura T (2001). Modelling seismic wave propagation in a two-dimensional cylindrical whole-earth model using the pseudospectral method. Geophys J Int 145: 689-708.

Zhang W and Chen X (2006). Traction image method for irregular free surface boundaries in finite difference seismic wave simulation. Geophys J Int 167: 337-353. 